\title{
Aromatic Oligoureas: Enforced Folding and Assisted Cyclization
}

\author{
Aimin Zhang, ${ }^{\dagger}$ Yaohua Han, ${ }^{\dagger}$ Kazuhiro Yamato, ${ }^{\dagger}$ Xiao Cheng Zeng, ${ }^{\ddagger}$ Bing Gong ${ }^{*}{ }^{\dagger}$ \\ ${ }^{\dagger}$ Department of Chemistry \\ University at Buffalo \\ The State University of New York, Buffalo, \\ New York 14260 \\ ${ }^{\ddagger}$ Department of Chemistry \\ University of Nebraska-Lincoln \\ Lincoln, Nebraska 68588
}

\section{Supporting Information}




\section{Scheme 1}

\section{Synthetic Procedures}<smiles>Cc1ccc([N+](=O)[O-])c(C)c1</smiles>

1<smiles>[R]OC(=O)c1cc(C(=O)O[R])c([N+](=O)[O-])cc1[N+](=O)[O-]</smiles>

4a $(72 \%)$

4b $(83 \%)$<smiles>Cc1cc(C)c([N+](=O)[O-])cc1[N+](=O)[O-]</smiles>

2

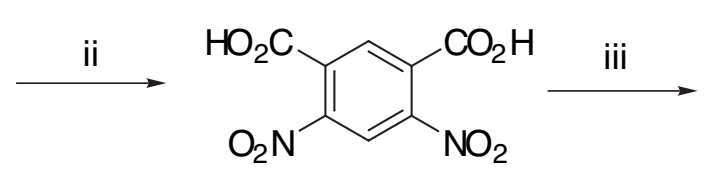

3

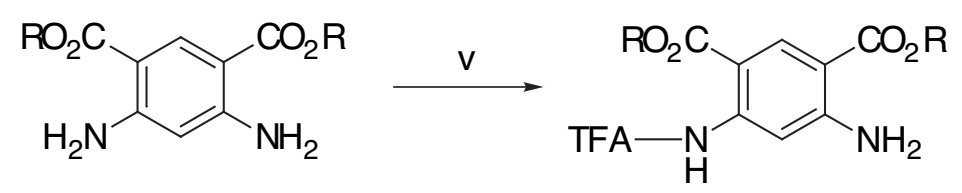

5a $(96 \%)$

5b $(92 \%)$ 6a $(75 \%)$

6b $(68 \%)$

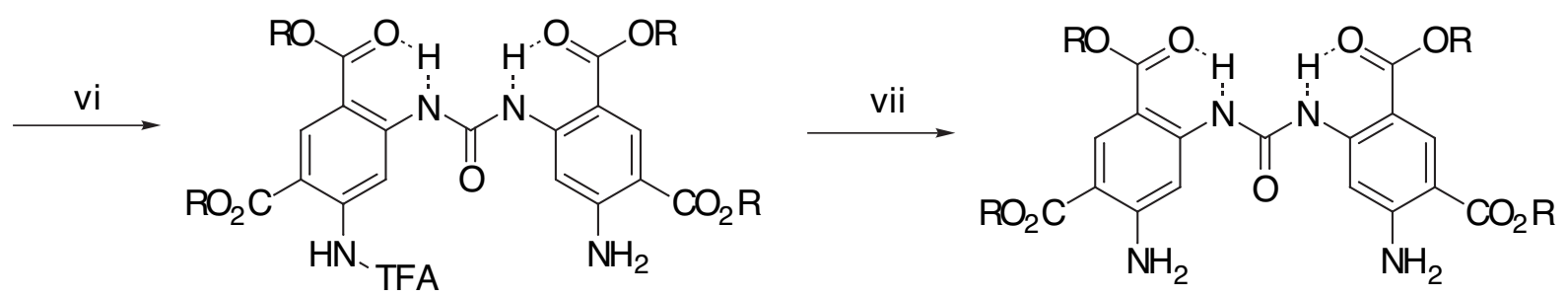

$7 a(25 \%)$

7 b $(40 \%)$

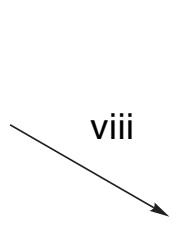

a $\mathrm{R}=5^{5}\left(\mathrm{C}_{8} \mathrm{H}_{17}\right)$

b $\mathrm{R}=\hat{\mathrm{Y}} \mathrm{O}\left(\mathrm{C}_{3} \mathrm{H}_{6} \mathrm{OC}_{5} \mathrm{H}_{11}\right)$

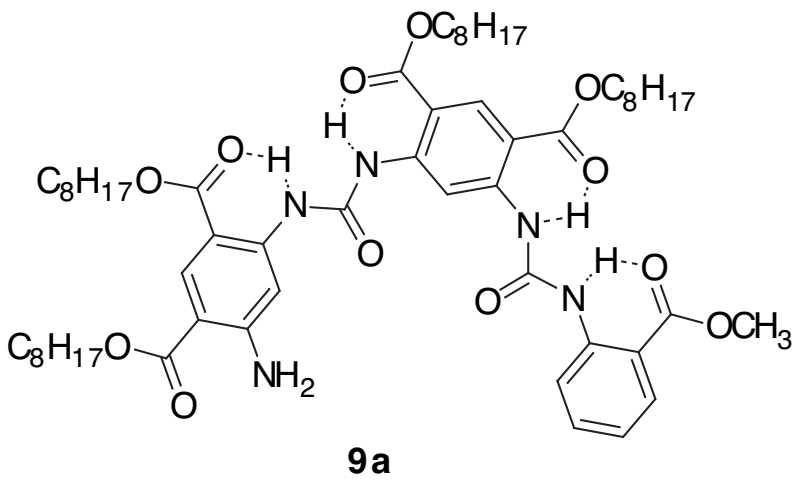

Reaction conditions: i) $70 \% \mathrm{HNO}_{3}$, conc. $\mathrm{H}_{2} \mathrm{SO}_{4}, 75 \%$; ii) conc. $\mathrm{H}_{2} \mathrm{SO}_{4}, \mathrm{CrO}_{3}, 70 \%$; iii) a. $\mathrm{SOCl}_{2}$, $\mathrm{CH}_{2} \mathrm{Cl}_{2}$; b. $\mathrm{C}_{8} \mathrm{H}_{17} \mathrm{OH}, \mathrm{Et}_{3} \mathrm{~N}, \mathrm{CH}_{2} \mathrm{Cl}_{2}$; iv) $\mathrm{H}_{2}, 10 \% \mathrm{Pd} / \mathrm{C}, \mathrm{CH}_{3} \mathrm{OH}$; v) $\left(\mathrm{CF}_{3} \mathrm{CO}\right)_{2} \mathrm{O}, \mathrm{Et}_{3} \mathrm{~N}, \mathrm{CH}_{2} \mathrm{Cl}_{2}$; vi) triphosgene, DMAP, $\mathrm{CH}_{2} \mathrm{Cl}_{2}$, 5, toluene; vii) $\mathrm{KHCO}_{3}, \mathrm{CH}_{2} \mathrm{Cl}_{2}, \mathrm{CH}_{3} \mathrm{OH}$; viii) a. toluene, 3carbomethoxyphenyl isocyanate; b. $\mathrm{KHCO}_{3}, \mathrm{CH}_{2} \mathrm{Cl}_{2}, \mathrm{CH}_{3} \mathrm{OH}, 18 \%$. 


\section{Scheme 2}<smiles>[R]OC(=O)c1cc(NC(=O)Nc2cc(N)c(C(=O)O[R])cc2C(=O)O)c(C(=O)O[R])cc1N</smiles>

a: $R=s^{5}$

b: $\mathrm{R}=\xi \overbrace{}^{\mathrm{O}}$

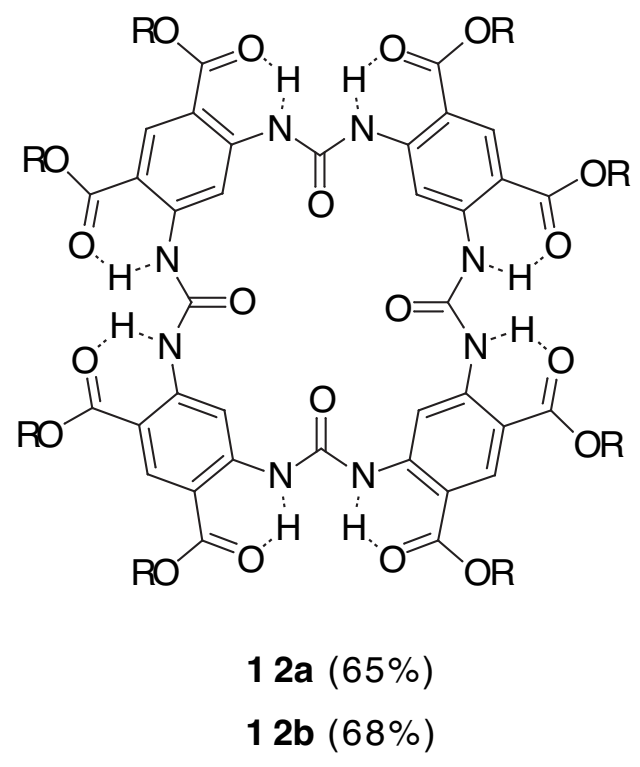

\section{Scheme 3}

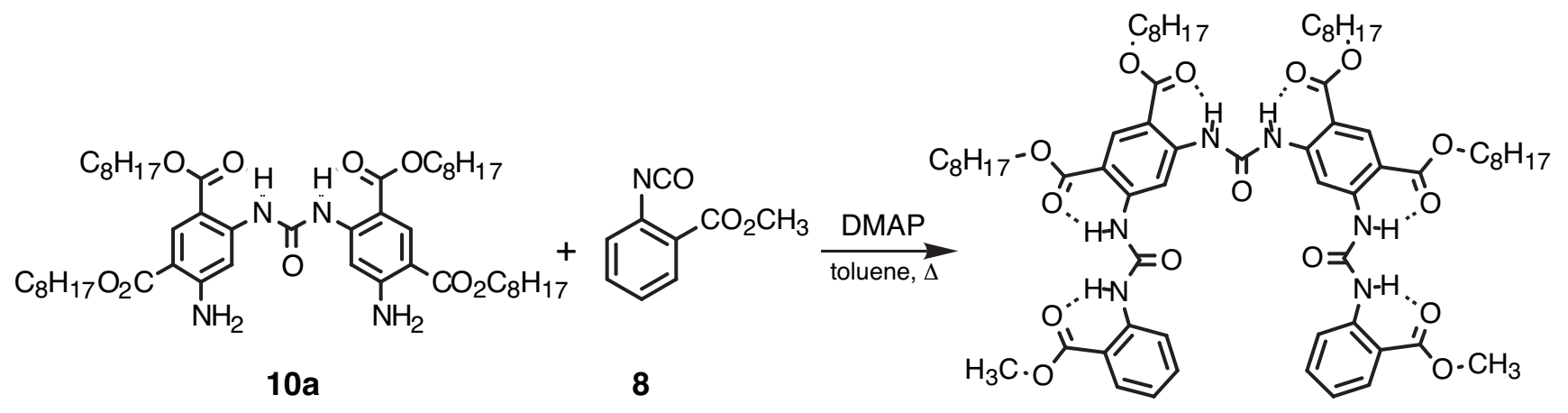

13a (74\%)

4,6-Dinitro-m-xylene (2). Compound $1(20 \mathrm{~mL}, 147.78 \mathrm{mmol})$ and concentrated $\mathrm{H}_{2} \mathrm{SO}_{4}(50 \mathrm{ml})$ were mixed and cooled in an ice water bath, to which $70 \% \mathrm{HNO}_{3}(10.3 \mathrm{ml}, 162.74 \mathrm{mmol})$ was added dropwise. The reaction was stirred for 1 hour, then diluted in $250 \mathrm{ml}$ ice-water. The precipitated product was collected by filtration, which was recrystallized from $\mathrm{CH}_{3} \mathrm{OH}$ to give pure product 2 (21.7 g, 75\%) as a yellow solid. ${ }^{1} \mathrm{H}-\mathrm{NMR}\left(400 \mathrm{MHz}, \mathrm{CDCl}_{3}\right) \delta 8.73(\mathrm{~s}, 1 \mathrm{H}), 7.37(\mathrm{~s}, 1 \mathrm{H}), 2.69(\mathrm{~s}, 6 \mathrm{H}) .{ }^{13} \mathrm{C}$ NMR (75.5 MHz, $\left.\mathrm{CDCl}_{3}\right) \delta 146.55,139.13,130.75,119.25,13.52$. 
4,6-Dinitroisophthalic acid (3). $\mathrm{CrO}_{3}(30 \mathrm{~g}, 300 \mathrm{mmol})$ and concentrated $\mathrm{H}_{2} \mathrm{SO}_{4}(100 \mathrm{ml})$ were mixed and cooled in an ice water bath. A solution of compound 2 (12 g, $61.2 \mathrm{mmol})$ in concentrated $\mathrm{H}_{2} \mathrm{SO}_{4}$ $(60 \mathrm{ml})$ was added to the reaction dropwise. The temperature was kept between $10 \sim 20{ }^{\circ} \mathrm{C}$. The reaction was stirred until the temperature dropped, then diluted in $500 \mathrm{ml}$ ice-water. The aqueous solution was collected by filtration, which was extracted with $150 \mathrm{ml}$ ether. Removal of ether gave the crude product, which was dissolved in water and extracted by $\mathrm{CH}_{2} \mathrm{Cl}_{2}$. The aqueous solution was extracted with $100 \mathrm{ml}$ ether, which was dried over anhydrous $\mathrm{Na}_{2} \mathrm{SO}_{4}$. Removal of ether gave the pure product 3 (10.9g, 70\%) as a yellow solid. ${ }^{1} \mathrm{H}-\mathrm{NMR}$ (400 $\left.\mathrm{MHz}, \mathrm{CDCl}_{3}, 5 \% \mathrm{DMSO}\right) \delta 8.32$ (s, $\left.1 \mathrm{H}\right), 8.23$ (s, 1H). ${ }^{13} \mathrm{C}$ NMR (75.5 MHz, $\mathrm{CDCl}_{3}, 5 \%$ DMSO) $\delta 164.07,148.70,132.01,131.45,119.04$.

Dioctyl 4,6-dinitroisophthalate (4a). Compound 3 (5.12 g, $20 \mathrm{mmol})$ and $\mathrm{SOCl}_{2}(10 \mathrm{ml}, 137 \mathrm{mmol})$ were mixed and refluxed until solid disappeared. Removal of $\mathrm{SOCl}_{2}$ gave solid acid chloride, which was dissolved in $10 \mathrm{ml} \mathrm{CH}_{2} \mathrm{Cl}_{2}$. The acid chloride solution was added dropwise to a solution of 1octanol $(5.7 \mathrm{~g}, 44 \mathrm{mmol})$ and $\mathrm{Et}_{3} \mathrm{~N}(7 \mathrm{ml}, 49 \mathrm{mmol})$ in $20 \mathrm{ml} \mathrm{CH}_{2} \mathrm{Cl}_{2}$ at $0^{\circ} \mathrm{C}$. The reaction was warmed to room temperature and stirred for 5 hours. The mixture was washed with diluted $\mathrm{HCl}$, dried over anhydrous $\mathrm{Na}_{2} \mathrm{SO}_{4}$. Removal of $\mathrm{CH}_{2} \mathrm{Cl}_{2}$ gave dark color oil, which was recrystallized from $\mathrm{CH}_{3} \mathrm{OH}$. Collect gray solid $4(6.92 \mathrm{~g}, 72 \%)$ by filtration. ${ }^{1} \mathrm{H}-\mathrm{NMR}\left(400 \mathrm{MHz}, \mathrm{CDCl}_{3}\right) \delta 8.43(\mathrm{~s}, 1 \mathrm{H}), 8.12(\mathrm{~s}$, $1 \mathrm{H}), 4.38(\mathrm{t}, 4 \mathrm{H}), 1.74(\mathrm{~m}, 4 \mathrm{H}), 1.30(\mathrm{~m}, 20 \mathrm{H}), 0.88(\mathrm{t}, 6 \mathrm{H}) .{ }^{13} \mathrm{C} \mathrm{NMR}\left(75.5 \mathrm{MHz}, \mathrm{CDCl}_{3}\right) \delta 167.95$, $155.25,133.07,131.98,119.65,66.83,32.93,30.9230 .21,29.05,26,91,23.55,15.02$.

Compound 4b. The same procedure as $\mathbf{4 a}$ and the yield is $83 \%$. Yield: $83 \%$; ${ }^{1} \mathrm{H}-\mathrm{NMR}(400 \mathrm{MHz}$, $\left.\mathrm{CDCl}_{3}\right) \delta 8.46(\mathrm{~s}, 1 \mathrm{H}), 8.14(\mathrm{~s}, 1 \mathrm{H}), 4.35(\mathrm{~m}, 4 \mathrm{H}), 3.72(\mathrm{~m}, 2 \mathrm{H}), 3.46(\mathrm{~m}, 4 \mathrm{H}), 1.71(\mathrm{~m}, 2 \mathrm{H}), 1.46(\mathrm{~m}$, 4H), $1.22(\mathrm{~d}, 6 \mathrm{H}), 0.90(\mathrm{~d}, 12 \mathrm{H}) .{ }^{13} \mathrm{C} \mathrm{NMR}\left(75.5 \mathrm{MHz}, \mathrm{CDCl}_{3}\right) \delta 162.8,149,132.4,131.4,120.3,76$, 70.2, 67.4, 39.1, 25.3, 22.8, 17. MS $m / z$ 551.3, 413.3.

Dioctyl 4,6-diaminoisophthalate (5a). A mixture of compound 4a (4.8 g, $10 \mathrm{mmol}), 10 \% \mathrm{Pd}-\mathrm{C}(0.72$ g, $15 \%$ weight of compound 4a) in $\mathrm{CH}_{3} \mathrm{OH} / \mathrm{CH}_{2} \mathrm{Cl}_{2}(80 \mathrm{ml} / 40 \mathrm{ml})$ was degassed and stirred under an atmosphere of $\mathrm{H}_{2}$ at room temperature for 4-6 hours. The mixture was then filtered and the solvent was removed under vacuum. The residue was dissolved in $\mathrm{CH}_{2} \mathrm{Cl}_{2}$, washed with dilute sodium bicarbonate solution and dried over anhydrous $\mathrm{Na}_{2} \mathrm{SO}_{4}$. Removal of solvent gave the crude product, which was washed with $\mathrm{CH}_{3} \mathrm{OH}$ to give pure product 5a $(4.04 \mathrm{~g}, 96 \%)$ as a white solid. ${ }^{1} \mathrm{H}-\mathrm{NMR}(500 \mathrm{MHz}$, 
$\left.\mathrm{CDCl}_{3}\right) \delta 8.54(\mathrm{~s}, 1 \mathrm{H}), 5.95(\mathrm{~s}, 4 \mathrm{H}), 5.74(\mathrm{~s}, 1 \mathrm{H}), 4.22(\mathrm{t}, 4 \mathrm{H}), 1.75(\mathrm{~m}, 4 \mathrm{H}), 1.44(\mathrm{~m}, 4 \mathrm{H}), 1.32(\mathrm{~m}$, $16 \mathrm{H}), 0.88(\mathrm{t}, 6 \mathrm{H}) .{ }^{13} \mathrm{C} \mathrm{NMR}\left(75.5 \mathrm{MHz}, \mathrm{CDCl}_{3}\right) \delta 166.83,152.97,131.91,108.35,101.91,65.29$, $32.61,30.79,29.98,28.96,26.15,22.96,14.98$.

Compound 5b. The same procedure as $\mathbf{5 a}$ and the yield is $92 \% .{ }^{1} \mathrm{H}-\mathrm{NMR}\left(500 \mathrm{MHz}, \mathrm{CDCl}_{3}\right) \delta 8.57(\mathrm{~s}$, 1H), 5.96(b, 4H), $5.74(\mathrm{~s}, 1 \mathrm{H}), 4.25(\mathrm{~m}, 4 \mathrm{H}), 3.73(\mathrm{~m}, 2 \mathrm{H}), 3.56(\mathrm{~m}, 4 \mathrm{H}), 1.71(\mathrm{~m}, 2 \mathrm{H}), 1.46(\mathrm{~m}, 4 \mathrm{H})$, $1.24(\mathrm{~d}, 6 \mathrm{H}), 0.90(\mathrm{~d}, 12 \mathrm{H}) .{ }^{13} \mathrm{C} \mathrm{NMR}\left(75.5 \mathrm{MHz}, \mathrm{CDCl}_{3}\right) \delta 167.4,154.6,138.1,102.5,98.8,76,73.6$, $68,67.4,66.1,39.1,25.3,22.8,17.6,16.1 . \mathrm{MS} m / z$ 475.3, 453.1.

Dioctyl 4,6-(mono-trifluoroacetyl)-diaminoisophthalate (6a). A solution of trifluoroacetic anhydride $(1.4 \mathrm{ml}, 10 \mathrm{mmol})$ in $70 \mathrm{ml} \mathrm{CH} \mathrm{Cl}_{2}$ was added to a solution of $\mathbf{5 a}(4.2 \mathrm{~g}, 10 \mathrm{mmol})$ and $\mathrm{Et}_{3} \mathrm{~N}(1.4 \mathrm{ml}, 10$ $\mathrm{mmol}$ ) in $30 \mathrm{ml} \mathrm{CH}_{2} \mathrm{Cl}_{2}$ over 5 hours period. The reaction was stirred for additional 2 hours, then was washed with $\mathrm{HCl} / \mathrm{H}_{2} \mathrm{O}$, and $\mathrm{NaHCO}_{3} / \mathrm{H}_{2} \mathrm{O}$, dried over $\mathrm{Na}_{2} \mathrm{SO}_{4}$. Removal of solvent gave crude product, which was purified by the chromatography on silica gel to gave pure 6a $(3.87 \mathrm{~g}, 75 \%)$ as a white solid. ${ }^{1} \mathrm{H}-\mathrm{NMR}\left(400 \mathrm{MHz}, \mathrm{CDCl}_{3}\right) \delta 12.64$ (s, 1H), 8.64 (s, 1H), 7.95 (s, 1H), 4.30 (m, 4H), 1.77 (m, 4H), $1.45(\mathrm{~m}, 4 \mathrm{H}), 1.31(\mathrm{~m}, 16 \mathrm{H}), 0.89(\mathrm{~m}, 6 \mathrm{H}) .{ }^{13} \mathrm{C} \mathrm{NMR}\left(75.5 \mathrm{MHz}, \mathrm{CDCl}_{3}\right) \delta 168.65,167.63,166.95$, $153.09,146.82,132.41,125.20,112.91,111.86,107.17,66.0765 .81,32.27,31.91,29.68,29.31$, $29.14,26.16,25.81,25.65,26.05,23.51,14.48$.

Compound $6 \mathbf{b}$. The same procedure as $6 \mathbf{a}$ and the yield is $68 \%$. ${ }^{1} \mathrm{H}-\mathrm{NMR}\left(500 \mathrm{MHz}, \mathrm{CDCl}_{3}\right) \delta 12.6(\mathrm{~s}$, $1 \mathrm{H}), 8.64(\mathrm{~s}, 1 \mathrm{H}), 7.96(\mathrm{~s}, 1 \mathrm{H}), 6.8(\mathrm{~b}, 2 \mathrm{H}), 4.25(\mathrm{~m}, 4 \mathrm{H}), 3.73(\mathrm{~m}, 2 \mathrm{H}), 3.56(\mathrm{~m}, 4 \mathrm{H}), 1.71(\mathrm{~m}, 2 \mathrm{H}), 1.46$ $(\mathrm{m}, 4 \mathrm{H}), 1.24(\mathrm{~d}, 6 \mathrm{H}), 0.90(\mathrm{~d}, 12 \mathrm{H}) .{ }^{13} \mathrm{C} \mathrm{NMR}\left(75.5 \mathrm{MHz}, \mathrm{CDCl}_{3}\right) \delta 168,166.7,155.5,142.9,136.7$, 116.9, 114.6, 113.6, 106.4, 76, 73.4, 70.6, 68, 39.1, 25.1, 22.7, 17.3, 16.6. MS m/z 571.0, 557.1, 421.2, 399.1 .

Compound 7a. A solution of $6 \mathbf{a}(2.07 \mathrm{~g}, 4 \mathrm{mmol})$ and DMAP $(0.98 \mathrm{~g}, 8 \mathrm{mmol})$ in $25 \mathrm{ml} \mathrm{CH}_{2} \mathrm{Cl}_{2}$ was added to the solution of triphosgene $(2.37 \mathrm{~g}, 8 \mathrm{mmol})$ in $10 \mathrm{ml} \mathrm{CH}_{2} \mathrm{Cl}_{2}$ dropwise. The reaction was refluxed for 2 hours, after which the solvent was removed and the residue was washed the residue with $5 \mathrm{ml}$ toluene. The solution of dioctyl 6-isocyanato-4-trifluoroacetylaminoisophthalate in toluene was collected by filtration, which was used directly for the next step without further purification. Compound $5 \mathbf{a}(1.68 \mathrm{~g}, 4 \mathrm{mmol})$ was dissolved in the above isocyanate in toluene. The reaction was 
refluxed for 3 days. Removal of toluene gave an oil, which was purified by the chromatography on silica gel to give the pure $7 \mathbf{a}(0.95 \mathrm{~g}, 25 \%)$ as a yellow solid. ${ }^{1} \mathrm{H}-\mathrm{NMR}\left(400 \mathrm{MHz}, \mathrm{CDCl}_{3}\right) \delta 12.48$ (s, 1H), 11.33 (s, 1H), $11.14(\mathrm{~s}, 1 \mathrm{H}), 9.97(\mathrm{~s}, 1 \mathrm{H}), 8.77$ (s, 1H), 8.62 (s, 1H), $8.00(\mathrm{~s}, 1 \mathrm{H}), 4.37(\mathrm{~m}, 4 \mathrm{H})$, $4.30(\mathrm{t}, 2 \mathrm{H}), 4.26(\mathrm{t}, 2 \mathrm{H}), 1.83(\mathrm{~m}, 8 \mathrm{H}), 1.45(\mathrm{~m}, 8 \mathrm{H}), 1.30(\mathrm{~m}, 32 \mathrm{H}), 0.89(\mathrm{~m}, 12 \mathrm{H}) .{ }^{13} \mathrm{C}$ NMR $(75.5$ $\left.\mathrm{MHz}, \mathrm{CDCl}_{3}\right) \delta 168.89,168.73,168.01,167.79,165.62,155.53,151.61,148.40,146.49,143.95$, 137.11, 135.48, 129.43,110.68, 110.16, 109.58, 105.69, 104.83, 104.55, 66.63, 66.54, 65.64, 65.15, $32.41,29.85,29.33,29.24,29.11,26.67,26.65,26.60,26.55,23.24,14.65$.

Compound 7b. The same procedure as $7 \mathbf{a}$ and the yield is $40 \%$. ${ }^{1} \mathrm{H}-\mathrm{NMR}\left(500 \mathrm{MHz}, \mathrm{CDCl}_{3}\right) \delta 12.35$ (s, 2H), 11.18 (s, 2H), 9.85 (s, 2H), 9.29 (s, 2H), 8.80 (s, 2H), 4.32 (m, 8H), 3.76(m, 4H), 3.52(m, 8H), $1.69(\mathrm{~m}, 4 \mathrm{H}), 1.44(\mathrm{~m}, 8 \mathrm{H}), 1.25(\mathrm{~d}, 12 \mathrm{H}), 0.84(\mathrm{~d}, 24 \mathrm{H}) .{ }^{13} \mathrm{C} \mathrm{NMR}\left(75.5 \mathrm{MHz}, \mathrm{CDCl}_{3}\right)$ 8166.6, 150.8, $148.3,144.4,110.8,110.6,109.8,73.5,68.9,68.3,39.3,25.5,23.1,17.6$. HRMS (MALDI-TOF) for $\mathrm{C}_{53} \mathrm{H}_{76} \mathrm{~F}_{6} \mathrm{~N}_{4} \mathrm{O}_{15} \mathrm{Na}^{+}$: Calcd. 1145.5; Found 1145.5.

Compound 9a. Compound 7a $(0.1 \mathrm{~g}, 0.11 \mathrm{mmol})$ and 3-carbomethoxyphenyl isocyanate $(0.12 \mathrm{~g}, 0.68$ mmol) were dissolved in $2 \mathrm{ml}$ toluene. The reaction was refluxed for 3 days. Removal of toluene gave an oil, which was purified by the chromatography on silica gel to give the pure $9 \mathrm{a}(20 \mathrm{mg}, 18 \%)$ as a white solid. ${ }^{1} \mathrm{H}-\mathrm{NMR}\left(500 \mathrm{MHz}, \mathrm{CDCl}_{3}\right) \delta 11.24$ (s, 1H), 11.11 (s, 21H), 10.89 (s, 1H), $9.84(\mathrm{~s}, 1 \mathrm{H})$, $8.75(\mathrm{~s}, 1 \mathrm{H}), 8.70(\mathrm{~d}, 1 \mathrm{H}), 8.62(\mathrm{~s}, 1 \mathrm{H}), 8.07(\mathrm{~s}, 1 \mathrm{H}), 8.01(\mathrm{~d}, 1 \mathrm{H}), 7.53(\mathrm{t}, 1 \mathrm{H}), 7.02(\mathrm{t}, 1 \mathrm{H}), 4.36(\mathrm{~m}$, 4H), $4.31(\mathrm{t}, 2 \mathrm{H}), 4.26(\mathrm{t}, 2 \mathrm{H}), 3.96(\mathrm{~s}, 3 \mathrm{H}), 1.80(\mathrm{~m}, 8 \mathrm{H}), 1.46(\mathrm{~m}, 8 \mathrm{H}), 1.33(\mathrm{~m}, 32 \mathrm{H}), 0.89(\mathrm{~m}, 12 \mathrm{H})$. ${ }^{13} \mathrm{C}$ NMR $\left(75.5 \mathrm{MHz}, \mathrm{CDCl}_{3}\right) \delta 169.61,168.84,168.65,168.25,167.87,165.63,155.48,151.95$, $151.80,148.06,147.88,146.78,142.99,140.35,137.06,135.82,135.17,131.28,122.02,120.74$, $114.89,108.11,105.50,104.90,104.58,66.06,65.56,65.09,52.98,32.43,30.29,29.86,29.21,26.65$, 23.25, 14.68. Anal. Calcd. for $\mathrm{C}_{58} \mathrm{H}_{85} \mathrm{~N}_{5} \mathrm{O}_{12}: \mathrm{C}, 66.71 ; \mathrm{H}, 8.20 ; \mathrm{N}, 6.71$. Found $\mathrm{C}, 66.53 ; \mathrm{H}, 8.05 ; \mathrm{N}$, 6.49 .

Compound 10a. $\mathrm{KHCO}_{3}(200 \mathrm{mg}, 2 \mathrm{mmole})$ was added to a solution of compound 7 (100 mg, 0.1 mmole) in $\mathrm{CH}_{2} \mathrm{Cl}_{2}$ and $\mathrm{CH}_{3} \mathrm{OH}(50 \mathrm{~mL}, 1: 1)$ and the mixture was stirred at room temperature overnight. The solid was removed by filtration. After removal of the solvent, the residue was purified by PTLC to furnish the diamine 10a (85 mg) in 95\% yield. ${ }^{1} \mathrm{H}-\mathrm{NMR}\left(500 \mathrm{MHz}, \mathrm{CDCl}_{3}\right) \delta 11.23(\mathrm{~s}$, 2H), $8.67(\mathrm{~s}, 2 \mathrm{H}), 7.86(\mathrm{~s}, 2 \mathrm{H}), 6.26(\mathrm{~b}, 4 \mathrm{H}), 4.31(\mathrm{~m}, 8 \mathrm{H}), 1.78(\mathrm{~m}, 8 \mathrm{H}), 1.45(\mathrm{~m}, 8 \mathrm{H}), 1.31(\mathrm{~m}, 32 \mathrm{H})$, 


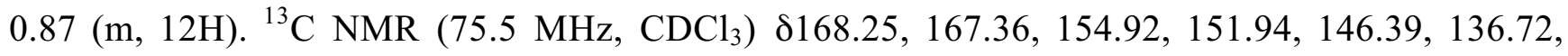
$104.98,104.18,103.76,65.14,64.65,31.94,29.39,28.82,28.74,26.19,26.16,22.77,14.20$. MS $m / z$ $867.8,866.9,635.2,557.1,421.1$.

Compound 10b. The same procedure as 10 a and the yield is $86 \% .{ }^{1} \mathrm{H}-\mathrm{NMR}\left(500 \mathrm{MHz}, \mathrm{CDCl}_{3}\right)$ $\delta 11.18(\mathrm{~s}, 2 \mathrm{H}), 8.67$ (s, 2H), 7.87 (s, 2H), 6.30 (b, 4H), $4.30(\mathrm{~m}, 8 \mathrm{H}), 3.74(\mathrm{~m}, 4 \mathrm{H}), 3.54(\mathrm{~m}, 8 \mathrm{H}), 1.69$ $(\mathrm{m}, 4 \mathrm{H}), 1.46(\mathrm{~m}, 8 \mathrm{H}), 1.25(\mathrm{~d}, 12 \mathrm{H}), 0.89(\mathrm{~d}, 24 \mathrm{H}) .{ }^{13} \mathrm{C} \mathrm{NMR}\left(75.5 \mathrm{MHz}, \mathrm{CDCl}_{3}\right) \delta 167.8,166.82$, $154.9,151.7,146.3,136.8,104.6,103.8,103.6,73.2,67.8,67.1,38.8,24.9,22.53$, 17.3. HRMS for $\mathrm{C}_{49} \mathrm{H}_{78} \mathrm{~N}_{4} \mathrm{O}_{13} \mathrm{Na}^{+}$: Calcd. 953.5482; Found 953.5463.

Compound 12a. To the solution of the diamine 10a (50 $\mathrm{mg}, 0.058 \mathrm{mmole})$ in dry toluene was added triphosgene (17 mg, $0.057 \mathrm{mmole})$, DMAP (14 mg, $0.11 \mathrm{mmole}$ ) and the mixture was refluxed in a sealed tube for two days. The reaction was quenched with water and extracted with $\mathrm{CH}_{2} \mathrm{Cl}_{2}$. The organic solution was dried with anhydrous $\mathrm{Na}_{2} \mathrm{SO}_{4}$. After removal of the solvent, the residue was purified by PTLC to furnish yellow solid 12a (33 mg, 65\%). ${ }^{1} \mathrm{H}-\mathrm{NMR}\left(500 \mathrm{MHz}, \mathrm{CDCl}_{3}\right) \delta 11.06(\mathrm{~s}$, $8 \mathrm{H}), 9.86(\mathrm{~s}, 4 \mathrm{H}), 8.47(\mathrm{~s}, 4 \mathrm{H}), 4.37(\mathrm{~m}, 16 \mathrm{H}), 1.86(\mathrm{~m}, 16 \mathrm{H}), 1.38(\mathrm{~m}, 80 \mathrm{H}), 0.92(\mathrm{~s}, 24 \mathrm{H}) .{ }^{13} \mathrm{C}$ NMR $\left(75.5 \mathrm{MHz} \mathrm{CDCl}_{3}\right) \delta 168.12,167.23,154.79,146.26,136.59,104.85,104.05,103.63,65.01,64.52$, 31.81, 29.25, 28.69, 28.61, 26.06, 26.03, 22.64, 14.07. HRMS (MALDI-TOF) for $\mathrm{C}_{100} \mathrm{H}_{152} \mathrm{~N}_{8} \mathrm{O}_{20} \mathrm{~K}^{+}$: Calcd. 1824.1; Found 1825.5.

Compound 12b. To a solution of diamine $10 \mathrm{~b}$ (50 $\mathrm{mg}, 0.054 \mathrm{mmole})$ in dry toluene was added triphosgene (17 mg, $0.057 \mathrm{mmole})$, DMAP (14 mg, $0.11 \mathrm{mmole})$ and the mixture was refluxed in a sealed tube for two days. The reaction was quenched with water and extracted with $\mathrm{CH}_{2} \mathrm{Cl}_{2}$. The organic solution was dried with anhydrous $\mathrm{Na}_{2} \mathrm{SO}_{4}$. After removal of the solvent, the residue was purified by PTLC to furnish yellow solid 12b (35 mg, 68\%). ${ }^{1} \mathrm{H}-\mathrm{NMR}\left(500 \mathrm{MHz}, \mathrm{CDCl}_{3}\right) \delta 11.07$ (s, $8 \mathrm{H}), 9.84(\mathrm{~s}, 4 \mathrm{H}), 8.84(\mathrm{~s}, 4 \mathrm{H}), 4.38(\mathrm{~m}, 16 \mathrm{H}), 3.75(\mathrm{~m}, 8 \mathrm{H}), 3.57(\mathrm{~m}, 16 \mathrm{H}), 1.85(\mathrm{~m}, 8 \mathrm{H}), 1.50(\mathrm{~m}$,

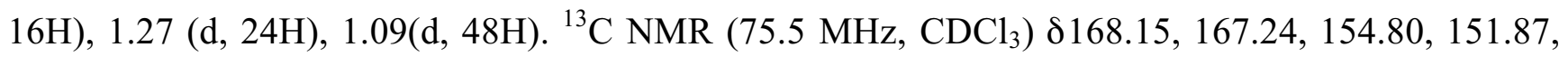
$146.28,136.62,104.96,104.16,103.72,65.04,64.56,31.82,29.25,28.73,28.65,26.08,26.05,22.64$, 14.07. HRMS (MALDI-TOF) for $\mathrm{C}_{100} \mathrm{H}_{152} \mathrm{~N}_{8} \mathrm{O}_{28} \mathrm{~K}^{+}$: Calcd. 1952.0; Found 1952.3. 
Compound 13a. Compound 10a $(250 \mathrm{mg}, 0.29 \mathrm{mmol}), 3$-carbomethoxyphenyl isocyanate (300 $\mathrm{mg}$, $1.69 \mathrm{mmol}$ ) and DMAP (70 $\mathrm{mg}, 0.57 \mathrm{mmol}$ ) were dissolved in $2 \mathrm{ml}$ toluene. The reaction was refluxed for 3 days. Removal of toluene gave an oil, which was purified by the chromatography on silica gel to give the pure tetramer 13a (260 mg, $74 \%$ ) as a white solid. ${ }^{1} \mathrm{H}-\mathrm{NMR}\left(500 \mathrm{MHz}, \mathrm{CDCl}_{3}\right) \delta 11.06(\mathrm{~s}$, 2H), $11.02(\mathrm{~s}, 2 \mathrm{H}), 10.85$ (s, 2H), $9.67(\mathrm{~s}, 2 \mathrm{H}), 8.74(\mathrm{~s}, 2 \mathrm{H}), 8.66(\mathrm{~d}, 2 \mathrm{H}), 7.99(\mathrm{~d}, 2 \mathrm{H}), 7.46(\mathrm{t}, 2 \mathrm{H})$, $6.98(\mathrm{t}, 2 \mathrm{H}), 4.35(\mathrm{~m}, 8 \mathrm{H}), 3.96(\mathrm{~s}, 6 \mathrm{H}), 1.81(\mathrm{~m}, 8 \mathrm{H}), 1.48(\mathrm{~m}, 8 \mathrm{H}), 1.34(\mathrm{~m}, 32 \mathrm{H}), 0.89(\mathrm{~m}, 12 \mathrm{H}) .{ }^{13} \mathrm{C}$ NMR (75.5 MHz, $\left.\mathrm{CDCl}_{3}\right)$ 8169.50, 168.21, 151.73, 151.39, 147.84, 147.48, 142.99, 135.55, 135.05, $131.14,121.88,120.58,114.72,109.29,108.49,108.39$, 66.04, 52.92, 32.42, 29.86, 29.21, 26.65, 23.24, 14.66. Anal. Calcd. for $\mathrm{C}_{67} \mathrm{H}_{92} \mathrm{~N}_{6} \mathrm{O}_{15}: \mathrm{C}, 65.88 ; \mathrm{H}, 7.59 ; \mathrm{N}, 6.88$. Found C, 65.64; H, 7.48; N, 6.66 . 


\section{Ab inito Calculation}

The density-functional calculation was carried out using the Gaussian 03 package. ${ }^{17}$ The geometry of each conformation was optimized by using the B3LYP/6-311+G(d,p) level of theory and basis set. Density-functional calculation shows that conformer $\mathbf{1 a}$ is the stable conformation whereas conformer $\mathbf{1 b}$ is $4.2915 \mathrm{kcal} / \mathrm{mol}$ higher in energy.

\section{Reference 13}

Gaussian 03, Revision C.02, Frisch, M. J.; Trucks, G. W.; Schlegel, H. B.; Scuseria, G. E.; Robb, M. A.; Cheeseman, J. R.; Montgomery, Jr., J. A.; Vreven, T.; Kudin, K. N.; Burant, J. C.; Millam, J. M.; Iyengar, S. S.; Tomasi, J.; Barone, V.; Mennucci, B.; Cossi, M.; Scalmani, G.; Rega, N.; Petersson, G. A.; Nakatsuji, H.; Hada, M.; Ehara, M.; Toyota, K.; Fukuda, R.; Hasegawa, J.; Ishida, M.; Nakajima, T.; Honda, Y.; Kitao, O.; Nakai, H.; Klene, M.; Li, X.; Knox, J. E.; Hratchian, H. P.; Cross, J. B.; Bakken, V.; Adamo, C.; Jaramillo, J.; Gomperts, R.; Stratmann, R. E.; Yazyev, O.; Austin, A. J.; Cammi, R.; Pomelli, C.; Ochterski, J. W.; Ayala, P. Y.; Morokuma, K.; Voth, G. A.; Salvador, P.; Dannenberg, J. J.; Zakrzewski, V. G.; Dapprich, S.; Daniels, A. D.; Strain, M. C.; Farkas, O.; Malick, D. K.; Rabuck, A. D.; Raghavachari, K.; Foresman, J. B.; Ortiz, J. V.; Cui, Q.; Baboul, A. G.; Clifford, S.; Cioslowski, J.; Stefanov, B. B.; Liu, G.; Liashenko, A.; Piskorz, P.; Komaromi, I.; Martin, R. L.; Fox, D. J.; Keith, T.; Al-Laham, M. A.; Peng, C. Y.; Nanayakkara, A.; Challacombe, M.; Gill, P. M. W.; Johnson, B.; Chen, W.; Wong, M. W.; Gonzalez, C.; and Pople, J. A.; Gaussian, Inc., Wallingford CT, 2004. 


\section{Energy-Minimized Structure of the Cyclic Tetramer (Methyl esters)}

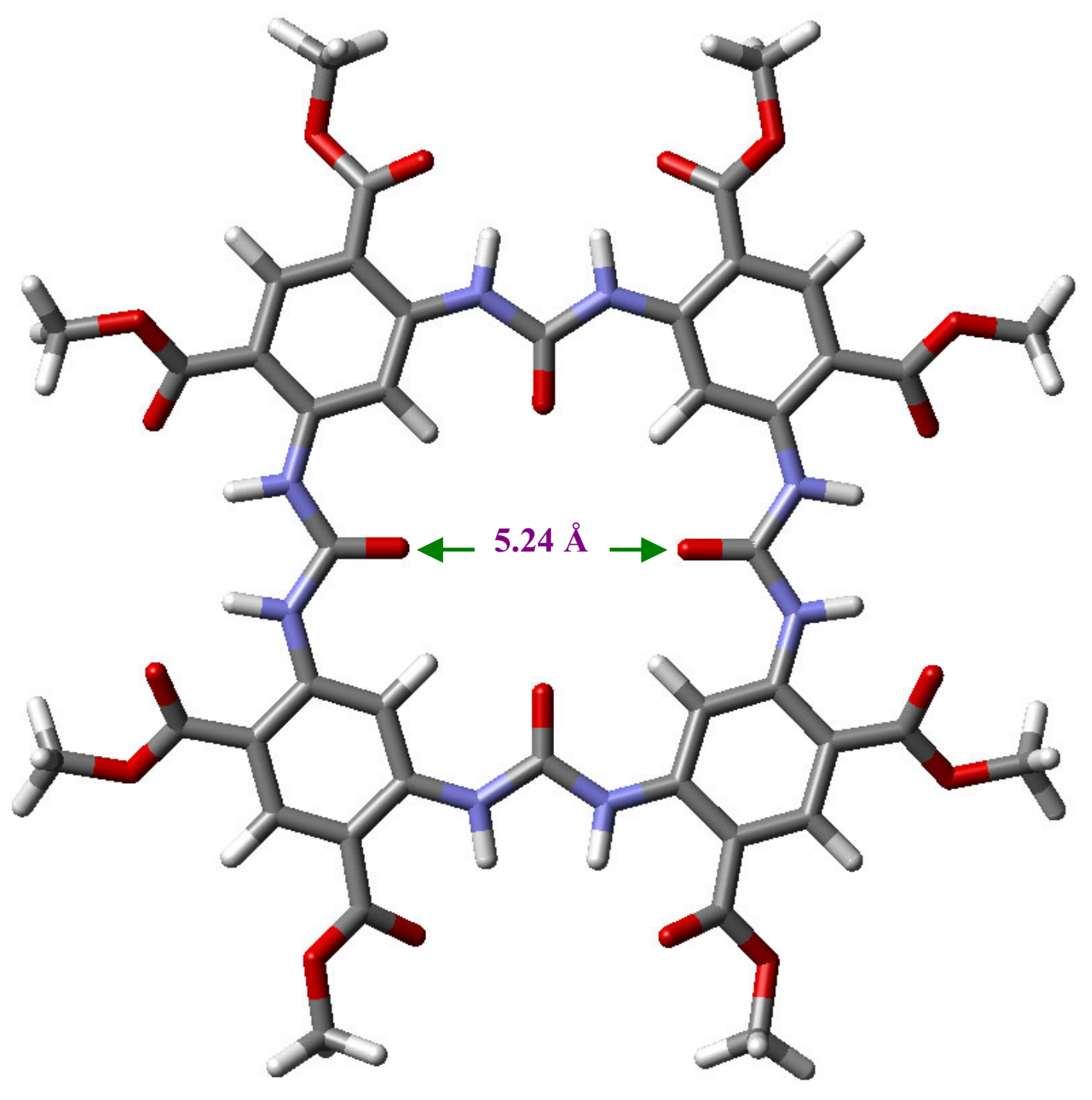




\section{MALDI Experiment}

MALDI Binding Experiments. The matrix solution of 9-nitroanthracene $\left(20 \mathrm{mM}, \mathrm{CHCl}_{3}\right)$ was prespotted $(2 \mathrm{uL})$ onto the steel sample target. Host-guest mixtures $\left(1 \mathrm{uL}, 1 \mathrm{mM}, 90 \% \mathrm{MeOH} / 10 \% \mathrm{CH}_{2} \mathrm{Cl}_{2}\right)$ were directly spotted onto the sample well and drawn back into the capillary tube and spotted again to insure an even matrix-sample mixture. The matrix-sample solution was air-dried on the target at ambient temperature. 